\title{
JQ1, an inhibitor of the epigenetic reader BRD4, suppresses the bidirectional MYC-AP4 axis via multiple mechanisms
}

\author{
SUNG KYUNG CHOI ${ }^{1}$, SEONG HWI HONG ${ }^{1}$, HYUK SOON KIM ${ }^{1}$, CHAN YOUNG SHIN $^{1,4}$, \\ SUK WOO NAM ${ }^{2}$, WAHN SOO CHOI ${ }^{1,4}$, JEUNG-WHAN HAN ${ }^{3}$ and JUENG SOO YOU ${ }^{1,4}$ \\ ${ }^{1}$ School of Medicine, Konkuk University, Seoul 143-701; ${ }^{2}$ Functional RNomics Research Center, College of Medicine, \\ Catholic University, Seoul 137-701; ${ }^{3}$ Research Center for Epigenome Regulation, School of Pharmacy, \\ Sungkyunkwan University, Suwon 440-746; ${ }^{4}$ Konkuk University Medical Centre, \\ Konkuk University, Seoul 143-701, Republic of Korea
}

Received August 18, 2015; Accepted October 6, 2015

DOI: $10.3892 /$ or.2015.4410

\begin{abstract}
Bromodomain and extra-terminal domain (BET) family proteins are representative epigenetic modulators that read acetylated lysine residues and transfer cellular signals. Recently, the BET protein inhibitor JQ1 was developed and has been extensively studied in many cancer cell types. We demonstrated that JQ1 effectively suppressed the MYC-AP4 axis and induced antitumorigenic effects by targeting a bidirectional positive loop between MYC and AP4 which was first proposed in the present study. MYC and AP4 are the direct targets of BRD4, as demonstrated by chromatin immunoprecipitation (ChIP) assay and BRD4 loss-of-function experiments. Although inhibition of the MYC/MAC dimer suppressed AP4, the efficacy of suppression was not as effective as BRD4 inhibition. Notably, AP4 loss-of-function studies demonstrated that AP4 is a major critical target of JQ1 and that MYC is a novel downstream target of AP4, as demonstrated by AP4 binding to the MYC promoter. Taken together, our results suggest that the epigenetic reader BRD4 is a key mediator of the activated MYC-AP4 axis, which supports the possibility that targeting BET protein is a novel therapeutic strategy for MYC-AP4 axis-activated cancers.
\end{abstract}

Correspondence to: Dr Jueng Soo You, Department of Biochemistry, School of Medicine, Konkuk University, 120 Neungdong-ro, Gwangjin-Gu, Seoul 143-701, Republic of Korea

E-mail: jsyou@kku.ac.kr

Abbreviations: BET, bromodomain and extra-terminal domain; ChIP, chromatin immunoprecipitation; BRD, bromodomaincontaining protein; bHLH, basic helix-loop-helix domain; AP4, activating enhancer binding protein 4; EMT, epithelial-mesenchymal transition

Key words: epigenetic reader BRD4, JQ1, MYC, AP4, breast cancer

\section{Introduction}

Epigenetic mechanisms regulate gene expression and establish cellular identity. Therefore, the absence of proper epigenetic marks contributes to the development of diseases including cancer (1). These epigenetic marks are recognized by reader proteins that interpret the chromatin information and signal other cellular components to facilitate chromatin remodeling $(2,3)$. Proteins in the bromodomain and extra-terminal domain (BET) family, including bromodomain-containing proteins (BRD)2, BRD3, BRD4, and BRDT (testis-specific), are well-known readers of acetyl lysine residues, which is not only the most abundant protein modification in cells but also is critical to chromatin structure. BETs regulate a variety of genes involved in the cell cycle, cell growth and inflammation (4). Thus, the targeting of these BETs has become an intense research issue in diverse therapeutic areas.

The proto-oncogene MYC is a transcription factor containing a basic helix-loop-helix (bHLH) domain that has been studied for more than 30 years. The biology of MYC has been studied extensively which has resolved various issues. MYC heterodimerizes with the bHLH protein MAX and binds to $\mathrm{CA}(\mathrm{C} / \mathrm{T}) \mathrm{GTG}$ of its target genes. These genes encompass a broad spectrum of functions, from cell cycle progression and cell growth to epithelial-mesenchymal transition (EMT) (5). Activating enhancer binding protein 4 (AP4) is considered a key mediator of mitogenicity for MYC and EMT, as well as for further metastasis (6,7). MYC directly binds to CACGTG motifs in the first intron of the AP4 and functions as an activator (7). AP4 protein belongs to the bHLH subfamily similar to MYC, although it recognizes symmetrical DNA sequences such as CAGCTG and exclusively forms homodimers $(7,8)$. The role of the MYC-AP4 axis in cell cycle regulation and tumorigenesis was only recently discovered (8). Although a number of studies have reported MYC amplification and/or overexpression in several types of cancers (9), targeting of the MYC or MYC-AP4 axis remains a distant challenge.

Recently, a potent, selective, small-molecule inhibitor of BET bromodomains, JQ1, was developed. The molecule antagonized BET bromodomain proteins during MYC-dependent transcription in several types of cancers, including multiple 
myeloma, acute myeloid leukemia and mixed lineage leukemia (10-12). However, a more recent study suggested that the efficacy of BET inhibitors is not always dependent on the downregulation of MYC (13). With characteristic targeting epigenetic signaling molecules, BET inhibitors may function differently in a cell context-dependent manner. Therefore, it is important to define the main target and underlying mechanism of BET inhibitors in different cellular contexts.

Breast cancer is the most frequent type of cancer diagnosed in women and is a representative heterogeneous disease that can be classified into several subtypes based on gene expression profiling and tumor histology (14). Approximately $75 \%$ of patients are hormone receptor-positive, and the treatment options for these patients have relied on anti-hormonal strategies. While most patients respond to endocrine agents and have shown improved overall survival, eventually, the majority of such patients become resistant to these agents (15). Development of a therapeutic strategy to combat this resistance and to effectively treat hormone receptor-negative breast cancer is crucial. Recent studies have shown that BET inhibitors are valuable candidates for overcoming resistance to endocrine agents by the suppression of MYC and PI3K signaling $(16,17)$.

In the present study, we determined whether JQ1, an inhibitor of the epigenetic reader BRD4, suppresses the MYC-AP4 axis in breast cancer. We found that JQ1 suppressed the MYC-AP4 axis in ER-positive and -negative breast cancer cell lines. We further studied the ER-negative breast cancer cell line MDA-MB-231 which is relative harder to target in the clinic. JQ1 downregulated the MYC-AP4 axis by direct inhibition of BRD4 binding to the MYC and AP4 promoters at early time-points and subsequently induced antitumorigenic effects, including cell cycle arrest, reduced wound healing, and soft agar colony formation. Using BRD4 loss-of-function experiments, we further demonstrated that MYC and AP4 are the direct targets of BRD4 inhibition. We found that loss of AP4 mimics almost all of the antitumorigenic effects of JQ1, suggesting that AP4 is a more sensitive target for BRD4-mediated inhibition of MDA-MB-231 cells. Of note, we demonstrated for the first time that MYC is a downstream target of AP4; hence, there is a bidirectional positive loop between MYC and AP4. Thus, inhibition of the MYC-AP4 axis can be better amplified by JQ1. Altogether, the results presented here demonstrate that the BET protein inhibitor is effective against MYC-AP4 axis-activated cancers and other diseases by targeting multiple points.

\section{Materials and methods}

RNA extraction and reverse transcription PCR. Total RNA was extracted using TRIzol reagent, digested with DNase I, and reverse transcribed using a High Capacity cDNA reverse transcription kit (Applied Biosystems). Amplification of cDNA was performed on a LightCycler ${ }^{\circledR} 480$ II using the LightCycler $^{\circledR}$ 480 SYBR Green I Master (both from Roche), using the recommended conditions. cDNAs were amplified using the following gene-specific primers: RT_MYC, 5'-CCCTGG TGCCGTGAAGC; 3'-TTGCTCGAGTTCTTTCTGCAGA; and RT_AP4, 5'-GCAGGCAATCCAGCACAT; 3'-GGAGGC GGTGTCAGAGGT; and RT_P21, 5'-GAGGCCGGGATG
AGTTGGGAGGAG; 3'-CAGCCGGCGTTTGGAGTGG TAGAA and RT_BRD4, 5'-AAGAAGCGCTTGGAAAA CAA; 3'-CAGGTTTTGCTGTCCCTGTT and RT_P53, 5'-CCCCTCCTGGCCCCTGTCATCTTC; 3'-GCAGCGCCT CACAACCTCCGTCAT; and RT_GAPDH, 5'-GAGTCA ACGGATTTGGTCGT; 3'-TGGAAGATGGTGATGGGATT.

Western blot analysis. Cells were lysed with RIPA buffer [150 mM NaCl, 1.0\% NP-40, 0.5\% sodium deoxycholate, $0.1 \%$ SDS, $50 \mathrm{mM}$ Tris- $\mathrm{HCl}$ ( $\mathrm{pH} 8.0)$ and protease inhibitors] and sonicated briefly ( $30 \%$ amplitude, $3 \mathrm{sec}$ ). Cell lysates were boiled in Laemmli sample buffer for $3 \mathrm{~min}$, and $30 \mu \mathrm{g}$ of each protein was subjected to SDS-PAGE. The protein concentration was measured using the Bradford protein assay. Antibodies against MYC (cat no. 9402S; Cell Signaling Technology or cat no. ab39688-100; Abcam), AP4 (cat no. HPA001912; Sigma-Aldrich), P21 (cat no. sc-756; Santa Cruz Biotechnology), BRD4 (cat no. 13440), and $\beta$-actin (cat no. 49675) (both from Cell Signaling Technology) were used. Proteins were transferred to polyvinylidene difluoride membranes; the membranes were blocked for $30 \mathrm{~min}$ in Trisbuffered saline (TBS) containing $0.1 \%$ Tween-20 and 5\% (w/v) dry skim milk powder, and incubated overnight with the primary antibodies (dilution ratio 1:1,000). The membranes were then washed with TBS-0.1\% Tween-20, incubated for $1 \mathrm{~h}$ with a secondary antibody (dilution ratio 1:10,000), and visualized using an enhanced chemiluminescence detection kit (Amersham Life Sciences) after exposure on an LAS-3000 image detection system (Fuji).

Chromatin immunoprecipitation assay (ChIP). ChiP assays were performed according to instructions from Upstate Biotechnology. For each ChIP, $100 \mu \mathrm{g}$ DNA, sheared by sonication (the DNA fragment size was 200-500 bp), was pre-cleared with protein A magnetic beads (cat no. 16-661; Upstate Biotechnology), and then $40 \mu \mathrm{g}$ of the DNA was precipitated by BRD4 (cat no. 13440; Cell Signaling Technology) or by AP4 (cat no. HPA001912; Sigma-Aldrich). After IP, the recovered chromatin fragments were subjected to real-time PCR. IgG control experiments were performed for all ChIPs and incorporated into the IP/Input (1\%) by presenting the results as (IP - IgG)/(Input - IgG). The following primers were used for amplification of the chromatin fragments by real-time PCR: ChIP_MYC promoter, 5'-ACACTAACATCC CACGCTCTG; 3'-GATCAAGAGTCCCAGGGAGA and ChIP_MYC enhancer 1, 5'-TGCTAATTGTGCCTCTCCTGT; 3'-ACTCCCAGCAAATCAGCCTA; and ChIP_MYC enhancer 2, 5'-GGTCGGACATTCCTGCTTTA; 3'-GAT ATGCGGTCCCTACTCCA and ChIP_MYC_promoter_ AP4 binding motif, 5'-CACTCTCCCTGGGACTCTTG, 5'-CACTCTCCCTGGGACTCTTG; 3'-GCGCCTACCATTT TCTTTTG and ChIP_AP4 promoter, 5'-GGGCGCTGC AAATAGTCCTT; 3'-CCGGGCGTGTGTATGTGTGT and ChIP_AP4 enhancer 1, 5'-CGCGACGTTTGTAAATTGC; 3'-CTCAGATCCCGAGGAAGGA and ChIP_AP4 enhancer 2, 5'-GAGGTGGGCGTTCTACGG; 3'-GGTTGGGCAGG AGTGTCTAC.

Cell cycle analysis. Cell cycle assays were performed using the Cycletest Plus DNA reagent kit (BD Biosciences), according 
to the manufacturer's instructions. The cell cycle profile of the cells was analyzed using a FACScan flow cytometer (BD Biosciences).

Soft agar colony-formation assay. The soft agar colonyformation assay was performed in 6-well plates. A bottom layer of agar $(0.5 \%)$ with enriched Dulbecco's modified Eagle's medium (DMEM) (final 10\% FBS) was initially poured. After the bottom agar solidified, MDA-MB-231 cells $\left(1.0 \times 10^{4}\right)$ were seeded on the top agar (0.3\%) with enriched DMEM (final $10 \%$ FBS) and incubated at $37^{\circ} \mathrm{C}$ for 21 days. The culture medium was replaced 1-2 times per week. Colonies were visualized by staining for $1 \mathrm{~h}$ with $0.005 \%$ crystal violet.

Wound-healing assay. Cells were grown to confluency in culture dishes and treated with $0.2 \mu \mathrm{M} \mathrm{JQ1}$. After overnight starvation in serum-free medium, the cell monolayers were scraped with a sterile micropipette tip. Initial gap widths $(0 \mathrm{~h})$ and residual gap widths at 6 and $24 \mathrm{~h}$ after wounding were determined by photomicrographs.

shRNA infection. shBRD4 and shAP4 constructs were purchased from Sigma-Aldrich. For lentiviral production, the Mission lentiviral packaging mix was used. Infected derivative cells stably expressing shRNA were selected in the presence of $1.25 \mu \mathrm{g} / \mathrm{ml}$ puromycin.

Statistical analysis. Results are expressed as mean \pm SEM. Most statistical comparisons were calculated by one-way ANOVA followed by Bonferroni's post hoc test using GraphPad Prism. A p-value $<0.05$ was considered to indicate a significant result.

\section{Results}

JQ1 suppresses the MYC-AP4 axis in breast cancer cell lines. To examine whether JQ1, a known BRD4 inhibitor, targets the MYC-AP4 axis in breast cancer cells, we used ER-negative MDA-MB-231 cells and ER-positive MCF7 cells as models. MDA-MB-231 and MCF7 cells were treated for $24 \mathrm{~h}$ with the indicated concentrations of JQ1 (Fig. 1). The morphology of the MDA-MB-231 cells became thinner with protrusions after treatment with increasing concentrations of JQ1 (Fig. 1A). In contrast, JQ1 had a modest effect on the morphology of the MCF7 cells (Fig. 1D). JQ1 suppressed the MYC-AP4 axis in a dose-dependent manner in the MDA-MB-231 and MCF7 cells, although the sensitivities differed (Fig. 1B, C, E and F). MCF7 cells were more sensitive than the MDA-MB-231 cells to suppression by JQ1; this sensitivity may have been caused by a slightly higher expression of MYC in the MDA-MB-231 cells (Fig. 1). Upregulation of P21 expression was accompanied by downregulation of MYC and AP4 (Fig. 1B, C, E and F), indicating that JQ1 targets the MYC-AP4 axis in both ER-negative and -positive breast cancer cell lines.

JQ1 suppresses the tumorigenicity of breast cancer cells. Based on a previous study showing that acute treatment with JQ1 inhibits BRD4 (11), we measured the suppression of the MYC-AP4 axis by JQ1 $(0.2 \mu \mathrm{M})$ at an early time-point in the MDA-MB-231 and MCF7 cells. Downregulation of MYC and
AP4 was observed after $6 \mathrm{~h}$ of treatment, and was accompanied by upregulation of P21 (Fig. 2A-D). Notably, the protein level of AP4 was almost completely abolished at $6 \mathrm{~h}$ post-treatment in both breast cancer cell lines (Fig. 2B and D), suggesting that AP4 is a highly sensitive and direct target of JQ1.

The MYC-AP4-P21 axis is responsible for G1 arrest in several types of cancer cells (8); therefore, we investigated the effect of JQ1 on the cell cycle. JQ1 treatment led to an increase in the percentage of cells in the G1 stage, from 64 to $85 \%$ in the MDA-MB-231 cells and from 55 to $86 \%$ in the MCF7 cells (Fig. 2E). Next, to assess additional antitumorigenic effects of JQ1, we performed a scratch wound-healing assay and a soft agar colony-formation assay using MDA-MB-231 cells. As shown in Fig. 2F and G, JQ1 efficiently reduced the wound-healing capacity and soft agar colony formation of the MDA-MB-231 cells, indicating that JQ1 has antitumor efficacy in breast cancer cells.

JQ1 negatively regulates the MYC-AP4 axis directly via suppression of BRD4 binding at the promoters of both genes. To determine whether downregulation of MYC and AP4 by JQ1 is associated with BRD4 binding, we performed chromatin immunoprecipitation (ChIP) with a BRD4 antibody in MDA-MB-231 cells (Fig. 3A and B). First, we measured BRD4 binding to the previously identified promoter, enhancer 1 and enhancer 2 of MYC (11). As shown in Fig. 3A, we found that BRD4 binding decreased early at the promoter and at enhancer 2 upon treatment with JQ1, but not at enhancer 1 . Next, we measured BRD4 binding to the previously identified promoter, enhancer 1 and enhancer 2 of AP4 (7). Reduced binding by BRD4 was detected, mainly in the promoter, rather than in the enhancers (Fig. 3B), suggesting that suppression of the MYC-AP4 axis is through the direct inhibition of BRD4 binding at the promoter of both genes.

To further establish that BRD4 is required for activation of the MYC-AP4 axis, we performed a loss-of-function study by generating stable BRD4-knockdown cells (Fig. 3C). We detected reduced expression of MYC and AP4 as well as an increase in P21 after BRD4 knockdown in the MDA-MB-231 cells (Fig. 3D), demonstrating that, in MDA-MB-231 cells, the MYC-AP4 axis is a direct target of the epigenetic reader, BRD4.

Inhibition of MYC/MAX heterodimerization suppresses AP4, but not as effectively as JQ1. To determine whether the activation of the MYC-AP4 axis occurs through the action of the MYC/MAX dimer, we used the small-molecule MYC inhibitor, 10058-F4. 10058-F4 prevents the binding of MYC/MAX dimers to targets and inhibits MYC-driven transformation $(18,19)$. While 10058-F4 did not alter MYC mRNA levels, a dose of $50 \mu \mathrm{M}$ downregulated AP4 mRNA. This dose was also sufficient to induce changes in cell morphology (Fig. 4A and C), suggesting that AP4 is a target of the MYC/MAX dimer. There was a partial decrease in the MYC and AP4 protein levels after treatment with 10058-F4 (Fig. 4B), and the effect of 10058-F4 on cell cycle arrest was mild when compared to the effect of JQ1 (Fig. 4D). These results suggest that inhibition of MYC/MAX dimerization in the activated MYC-AP4 axis is not as effective as BRD4 inhibition, presumably due to a smaller downregulation of AP4. 
A MDA-MB-231

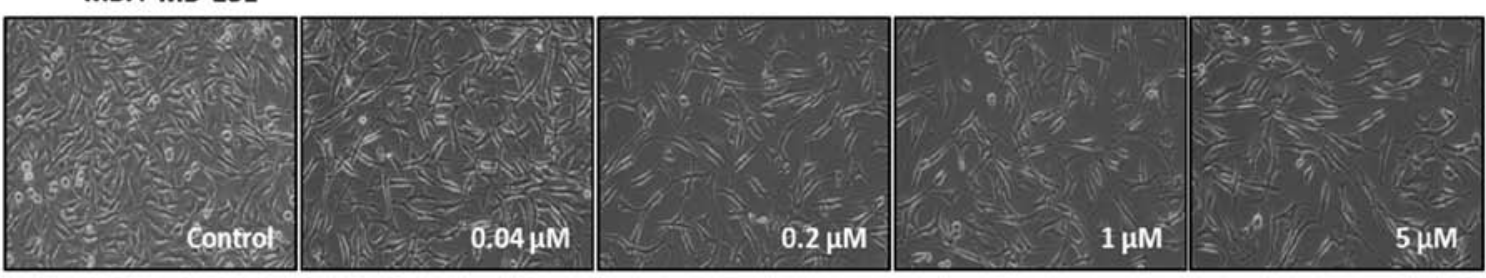

B

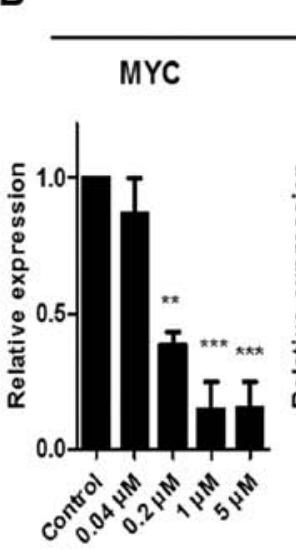

MDA-MB-231

AP4

P21
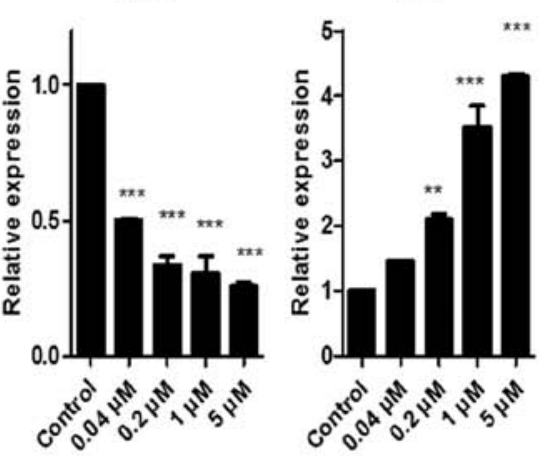

C

MDA-MB-231

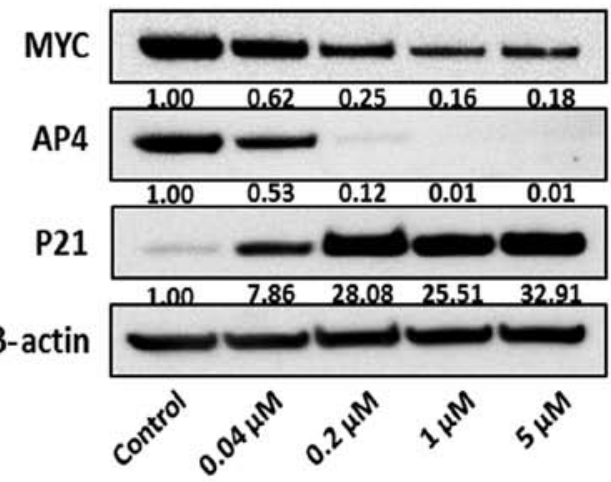

D
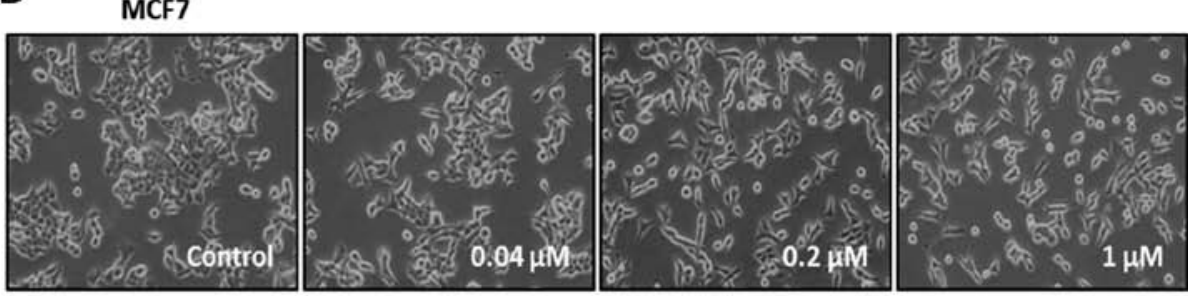

E

MCF7

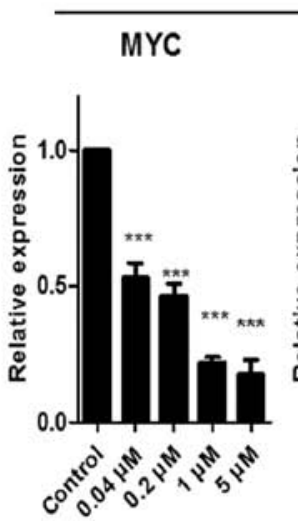

AP4

P21

\section{$\mathbf{F}$}

MCF7

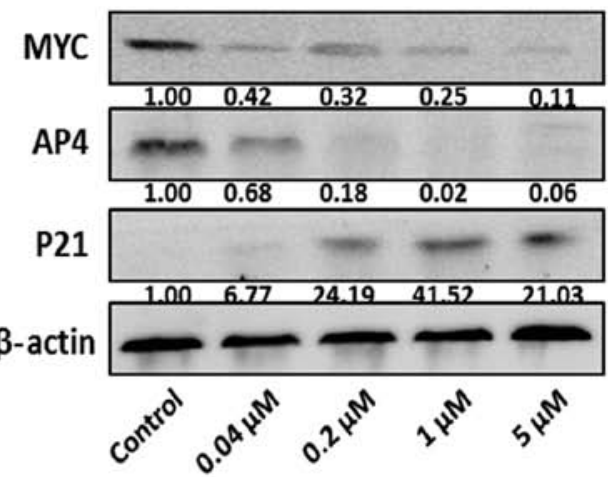

Figure 1. JQ1 suppresses the MYC-AP4 axis in ER-negative and -positive breast cancer cells. MDA-MB-231 (A-C) and MCF7 (D-F) cells were treated with increasing concentrations of JQ1 for $24 \mathrm{~h}$. (A and D) Cell morphology at a magnification of x100. (B and E) MYC, AP4, and P21 mRNA levels were determined by real-time PCR. Data are presented as the mean \pm SEM $\left({ }^{* *} \mathrm{P}<0.01,{ }^{* * *} \mathrm{P}<0.001\right.$ vs. control). (C and F) MYC, AP4, and P21 protein levels were analyzed by western blot analysis. Results shown are representative of three independent experiments.

Knockdown of AP4 reveals a bidirectional, positive loop between MYC and AP4 and the underlying mechanism by which suppression of the MYC-AP4 axis is synergized after treatment with JQ1. To determine whether AP4 is the major target of BRD4 inhibition, we generated a stable AP4-knockdown cell line using shRNA in the MDA-MB-231 cells. We then analyzed the mRNA and protein expression of
AP4, MYC, and P21 (Fig. 5A and B). We verified complete loss of AP4 by western blot analysis (Fig. 5B) and found that loss of AP4 mimicked most of the effects of treatment with JQ1, including suppression of the MYC-AP4 axis and subsequential induction of P21 (Fig. 5A and B), changes in cell morphology (Fig. 5C), cell cycle arrest (Fig. 5D), and reduced soft agar colony formation (Fig. 5E). These results suggest 
A

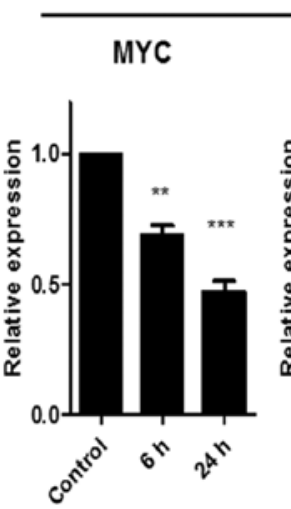

C

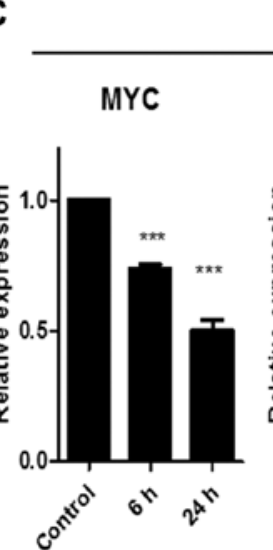

MDA-MB-231

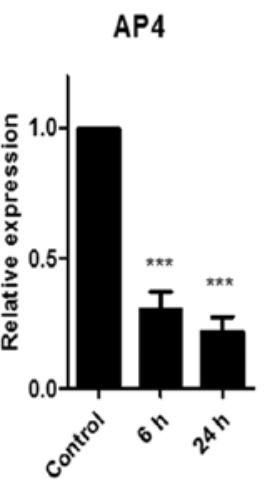

MCF7

AP4

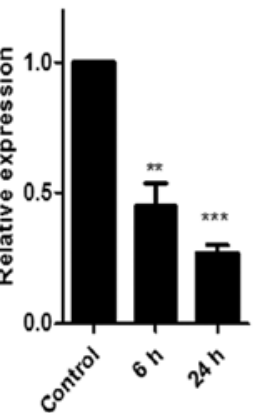

P21

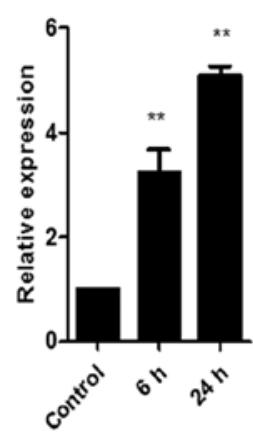

B
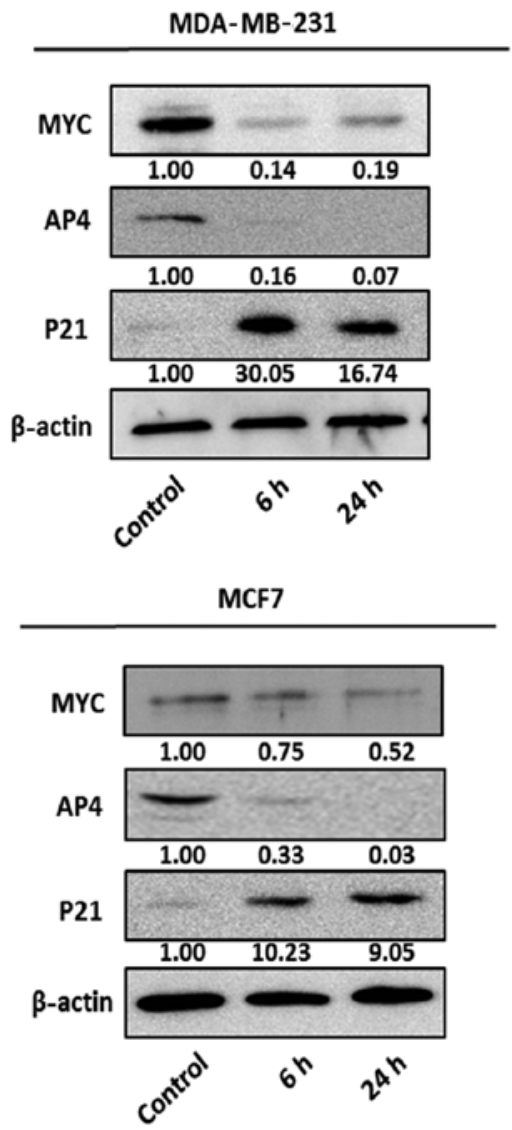

D
E

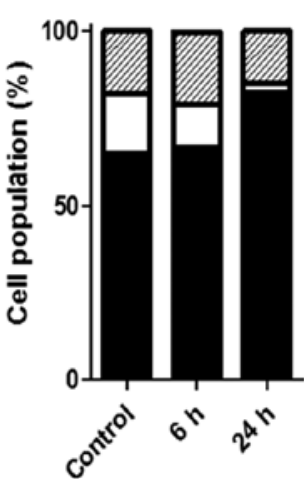

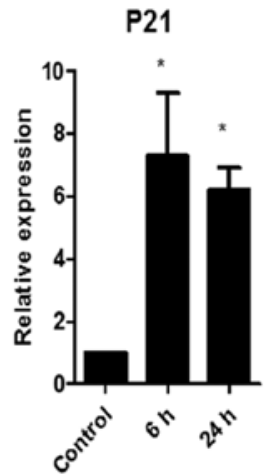

$$
1
$$

$\mathbf{F}$

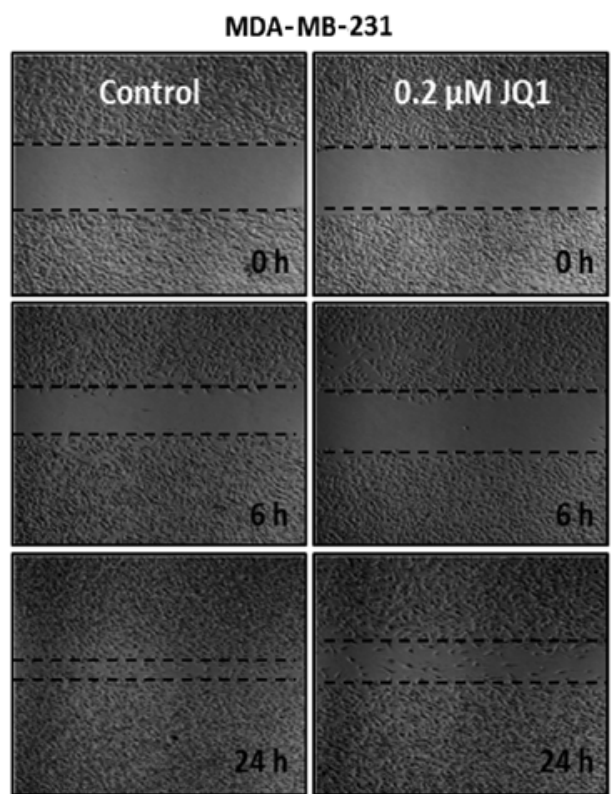

Figure 2. JQ1 induces antitumorigenic effects. MDA-MB-231 (A and B) and MCF7 (C and D) cells were treated with $0.2 \mu \mathrm{M}$ JQ1 for 6 and $24 \mathrm{~h}$. (A and C) MYC, AP4, and P21 mRNA levels were determined by real-time PCR. Data are presented as the mean $\pm \mathrm{SEM}$. $\left({ }^{*} \mathrm{P}<0.05,{ }^{* *} \mathrm{P}<0.01,{ }^{* * *} \mathrm{P}<0.001 \mathrm{vs}\right.$. control). (B and D) MYC, AP4, and P21 protein levels were analyzed by western blot analysis. Results shown are representative of three independent experiments. (E) DNA content was determined by flow cytometry. (F) MDA-MB-231 cells were treated with $0.2 \mu \mathrm{M}$ of JQ1 and subjected to wound healing assay. (G) Soft agar colony-formation assay was performed with and without JQ1 treatment. Results shown are representative of three independent experiments.

that AP4 is a major critical target of JQ1 in MDA-MB-231 cells. In addition, we confirmed that the expression of AP4 was reduced by JQ1 treatment in other cancer cell lines, including cell lines derived from the liver, colon and the esophagus (data not shown), suggesting that AP4 may be a general target of JQ1. 
A

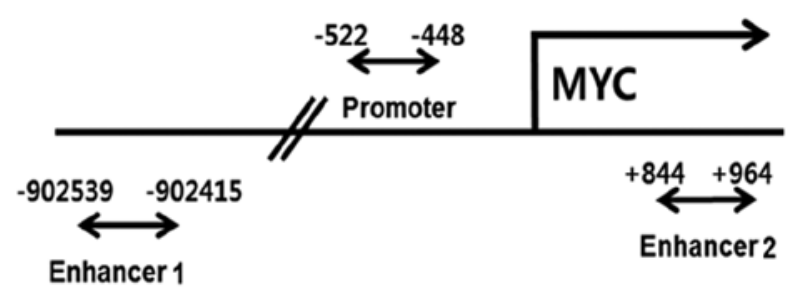

Enhancer 1
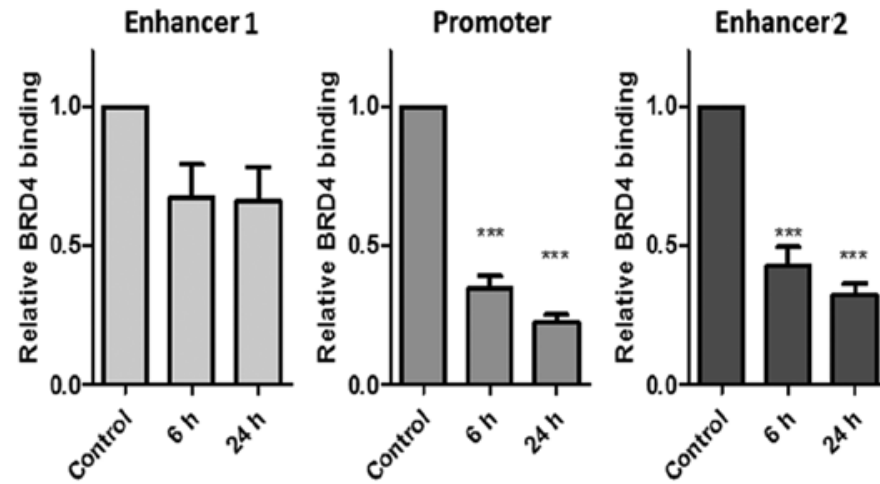

B
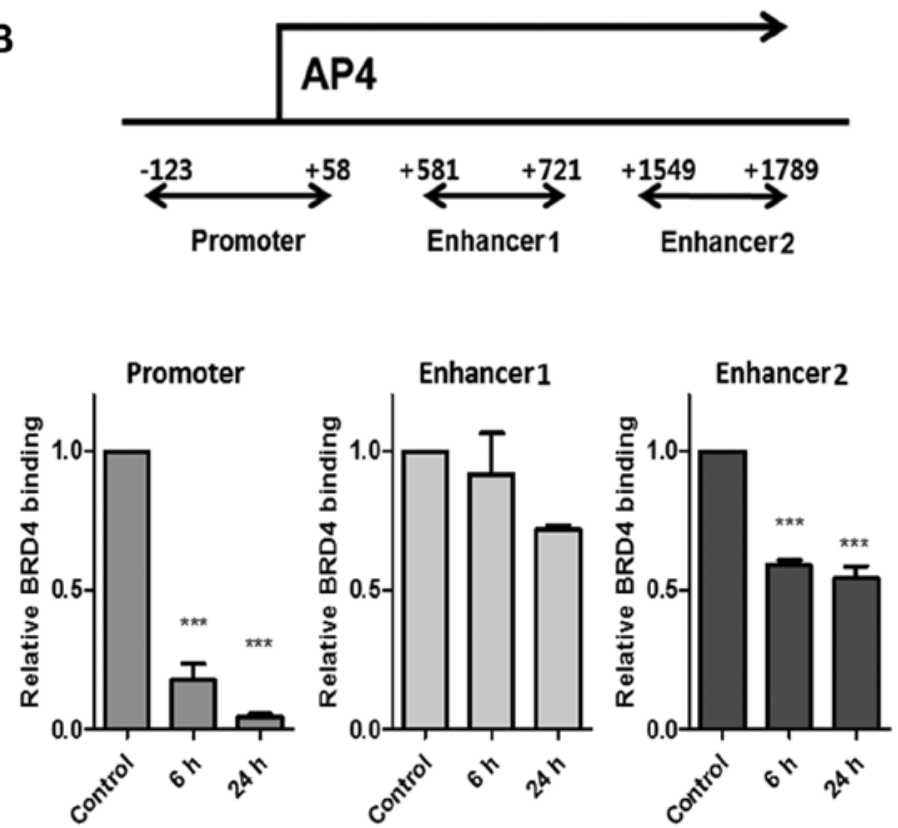

C

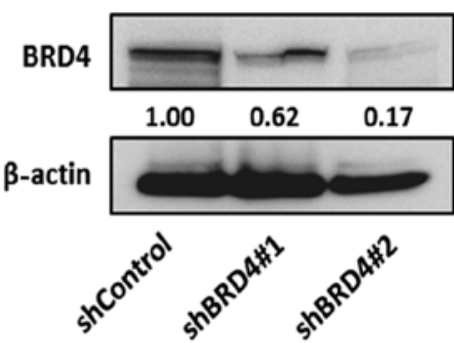

D
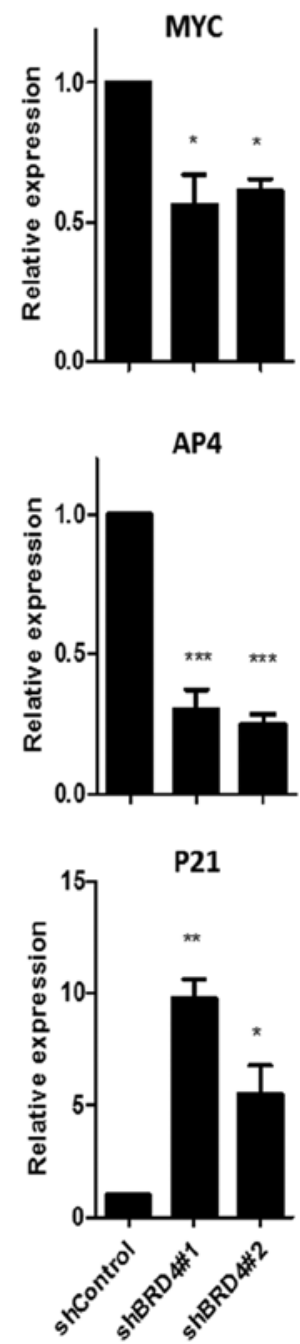

Figure 3. JQ1 downregulates the MYC-AP4 axis directly via suppression of BRD4 binding at the promoters of both genes. (A) Schematic diagram of the MYC promoter and enhancers. Chromatin was immunoprecipitated with anti-BRD4 antibody, and BRD4 binding at the MYC regulatory regions was analyzed by real-time PCR. Data are presented as the mean \pm SEM $\left({ }^{* * *} \mathrm{P}<0.001\right.$ vs. control). (B) Schematic diagram of the AP4 promoter and enhancers. Chromatin was immunoprecipitated with anti-BRD4 antibody and BRD4 binding at the AP4 regulatory regions was analyzed by real-time PCR. Data are presented as the mean \pm SEM $\left({ }^{* * * *} \mathrm{P}<0.001\right.$ vs. control). (C) The efficacy of BRD4 knockdown was determined by western blot analysis. (D) MYC, AP4, and P21 mRNA levels were determined by real-time PCR after loss of BRD4. Data are presented as the mean \pm SEM $\left({ }^{*} \mathrm{P}<0.05,{ }^{* * *} \mathrm{P}<0.01,{ }^{* * * *} \mathrm{P}<0.001\right.$ vs. control).

Unexpectedly, we observed a decrease in MYC upon AP4 knockdown (Fig. 5A and B). AP4 is known to be a direct target of MYC (6,7); however, to the best of our knowledge, it has not been reported that MYC is a downstream target of AP4. To confirm that MYC is a downstream target of AP4 and that there is a bidirectional positive loop between MYC and AP4, we performed a ChIP assay using the AP4 antibody following JQ1 treatment. We identified an AP4 binding motif
(CAGCTG) within the MYC promoter. We detected AP4 binding at this site, and this binding decreased upon treatment with JQ1 (Fig. 5F), demonstrating that MYC is a downstream target of AP4. Taken together, these data suggest that suppression of the MYC-AP4 axis by JQ1 is synergized through multiple mechanisms, including inhibition of BRD4 binding at the MYC and AP4 promoters, followed by secondary inhibition of a bidirectional loop between MYC and AP4. 
A

MYC

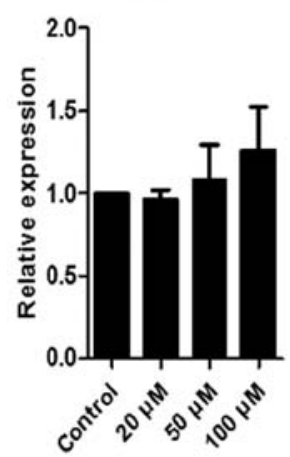

AP4

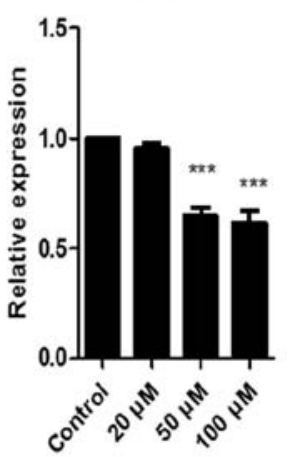

P21

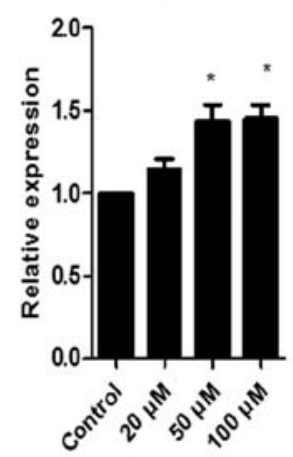

B

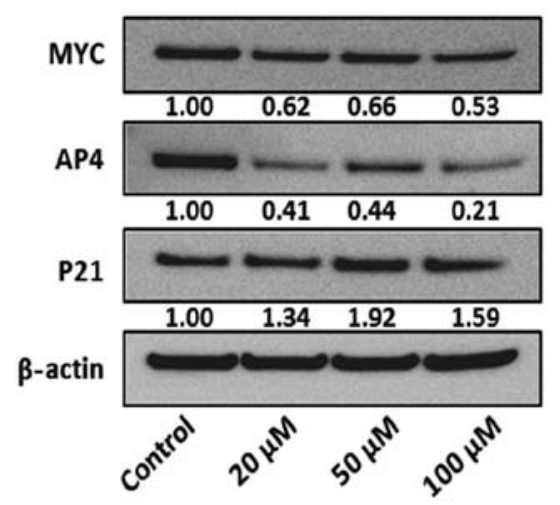

C

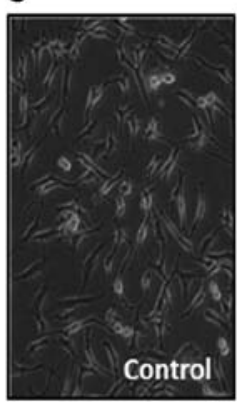

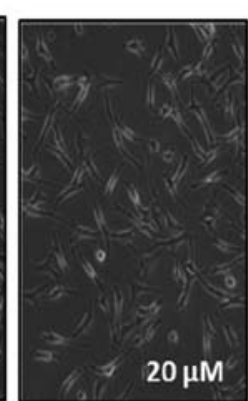

$20 \mu \mathrm{M}$

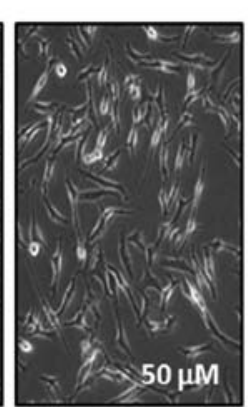

D
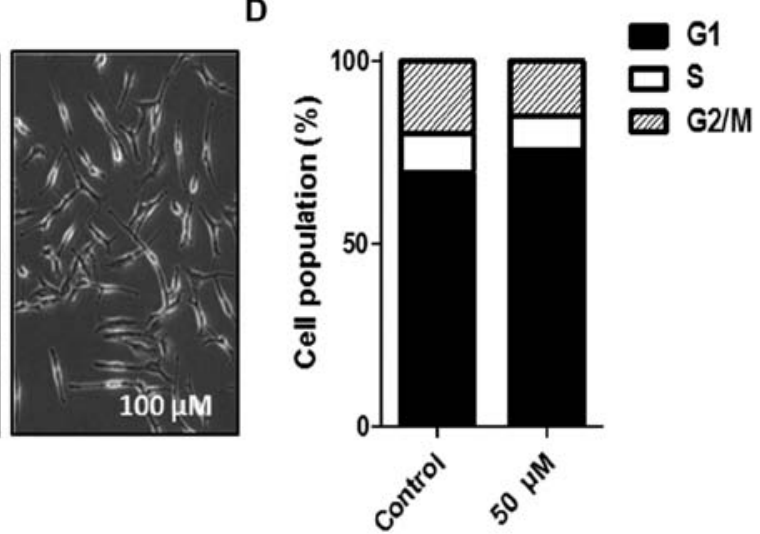

Figure 4. AP4 is a target of the MYC/MAX dimer while the MYC/MAX heterodimerization inhibitor 10058-F4 is not as effective as JQ1. MDA-MB-231 cells were treated with increasing concentrations of 10058-F4 for 24 h. (A) MYC, AP4, and P21 mRNA levels were determined by real-time PCR. Data are presented as the mean \pm SEM $\left({ }^{*} \mathrm{P}<0.05,{ }^{* *} \mathrm{P}<0.01,{ }^{* * *} \mathrm{P}<0.001\right.$ vs. control). (B) MYC, AP4, and P21 protein levels were analyzed by western blot analysis. (C) Cell morphology micrographs at a magnification of $x 100$. (D) DNA content was determined by flow cytometry. Results shown are representative of three independent experiments.

\section{Discussion}

Here, for the first time, we demonstrated that the inhibitor of the epigenetic reader BRD4, JQ1, effectively suppressed the MYC-AP4 axis in breast cancer cells by targeting a bidirectional, positive loop between MYC and AP4 (Fig. 5G). JQ1 inhibited BRD4 binding mainly at the promoters for MYC and AP4 promoters, rather than at the enhancer sites. We further demonstrated that MYC and AP4 are direct targets of BRD4 by generating stable BRD4-knockdown breast cancer cell lines. We found that the suppressive effect of an inhibitor of MYC/MAX dimerization on the activated MYC-AP4 axis was not as effective as inhibition of BRD4, which presumably acts through the mild downregulation of AP4. Our AP4 loss-of-function study demonstrated that AP4 is a major critical target of JQ1 and that MYC is a novel target of AP4, which was further supported by an anti-AP4 ChIP assay. Downregulation of MYC-AP4 by inhibition of BRD4 induced antitumorigenic effects, including cell cycle arrest, reduced wound healing and soft agar colony formation. Collectively, our results suggest that the epigenetic reader BRD4 is a key mediator of the overexpression of MYC and AP4. These findings have important implications for the treatment of MYC-AP4 axis-activated cancers.

Inhibitors of the epigenetic reader BET family, including JQ1 and I-BET, have emerged as promising therapeutic drugs for cancers, inflammation and obesity (20). The underlying mechanisms of the effects of these small molecules often involve binding of these drugs to the bromodomain of BET proteins, and the process is completed upon lysine acetylation of histones. The molecules, therefore, suppress target gene expression involved in tumorigenesis or the inflammatory response $(4,20)$. MYC is the primary target in several cancers $(11,12,21)$, and more recently, the underlying mechanism was proposed to involve inhibition by JQ1 of MYC by disrupting super-enhancers (22). Super-enhancers are defined as large clusters of enhancers that determine cellular identity $(23,24)$. JQ1 was found to lead to preferential loss of BRD4 at super-enhancers of MYC in multiple myeloma (22); however, it is not clear whether MYC always has a super-enhancer in different cellular contexts. Indeed, our results demonstrated that JQ1 inhibited BRD4 binding to the MYC promoter and enhancer 2, but not to enhancer 1 (Fig. 3A). Thus, the detailed molecular mechanism of BET protein inhibitors requires further investigation.

AP4 is known to be a MYC-inducible repressor of P21 (7); however, it has not been previously reported that MYC is a downstream target of AP4. Here, we demonstrated, for the first time, that there is a bidirectional positive loop between MYC and AP4 (Fig. 5). As shown in Fig. 5, AP4 binds to the MYC promoter. Binding by AP4 is reduced by JQ1 treatment, and 
A

MYC

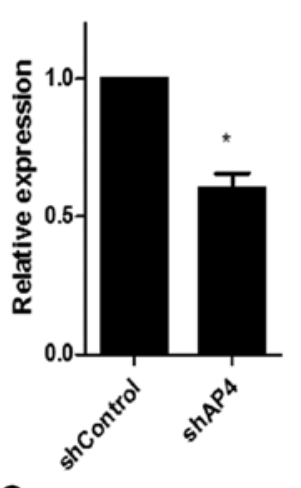

C
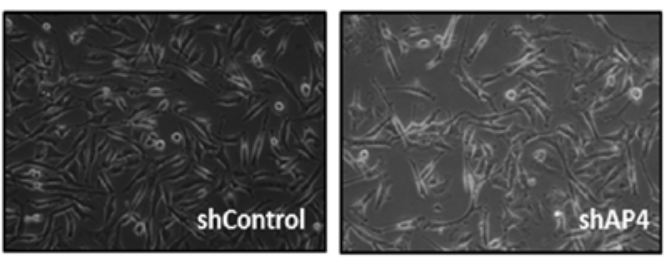

E

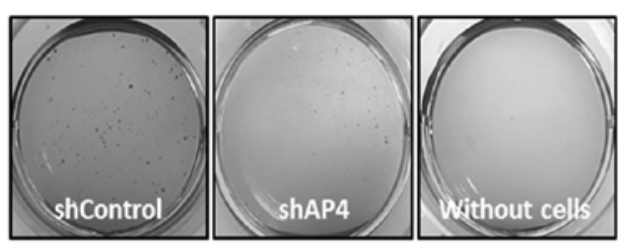

P21

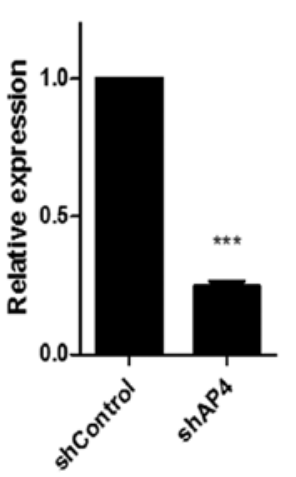

B
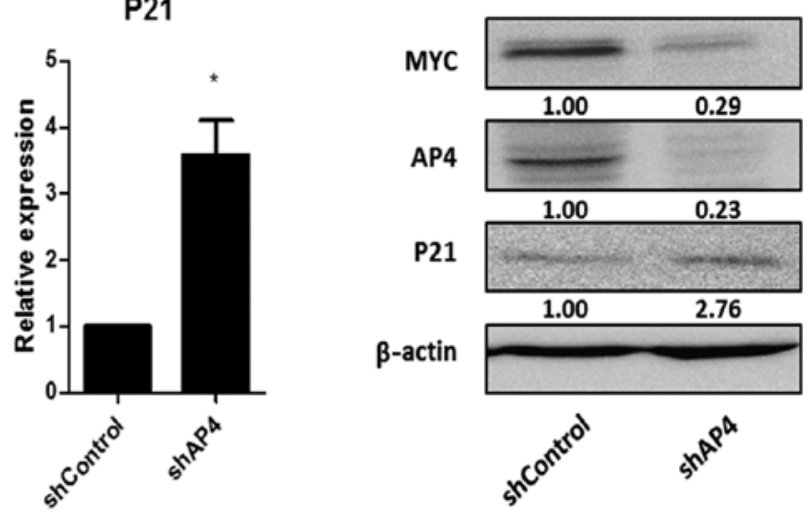

$\mathbf{F}$

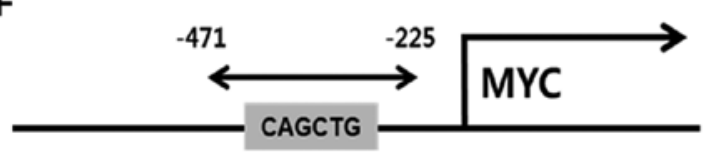

AP4 binding motif

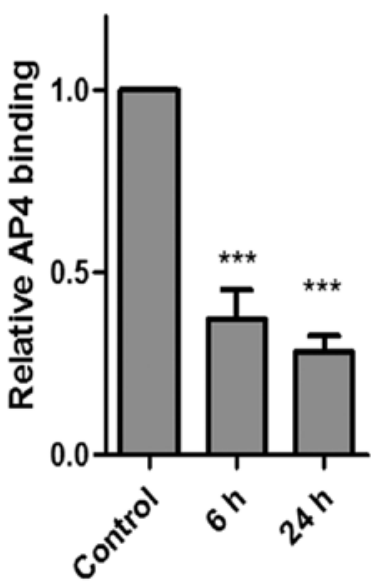

G

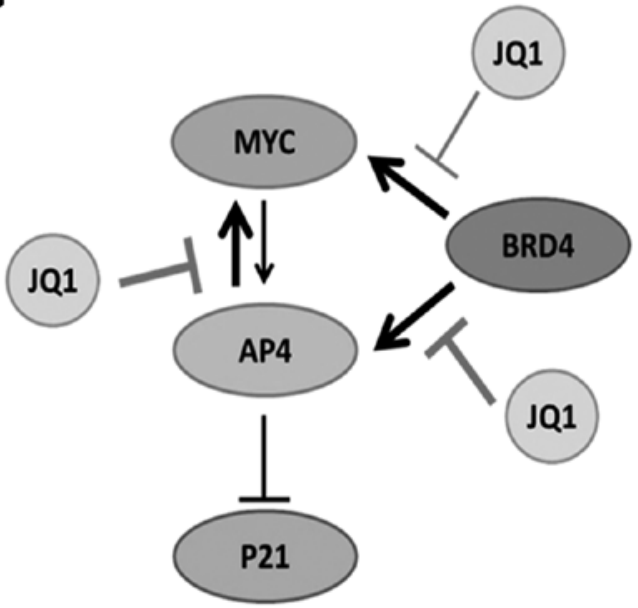

Figure 5. Knockdown of AP4 reveals a bidirectional positive loop between MYC and AP4 and the underlying mechanism through which suppression of the MYC-AP4 axis is synergized after JQ1 treatment. Stably infected AP4-knockdown MDA-MB-231 cells were selected with puromycin. (A) MYC, AP4, and P21 mRNA levels were determined by real-time PCR. Data are presented as the mean $\pm \mathrm{SEM}\left({ }^{*} \mathrm{P}<0.05,{ }^{* *} \mathrm{P}<0.01,{ }^{* * * *} \mathrm{P}<0.001\right.$ vs. control). (B) MYC, AP4, and P21 protein levels were analyzed by western blot analysis. Results shown are representative of three independent experiments. (C) Cell morphology micrographs at a magnification of $\mathrm{x} 100$. Results shown are representative of three independent experiments. (D) DNA content was determined by flow cytometry. Results shown are representative of three independent experiments. (E) Soft agar colony-formation assay was performed after stable knockdown of AP4. (F) Schematic diagram of the AP4 binding motif on the MYC promoter. Chromatin was immunoprecipitated with the anti-AP4 antibody, and AP4 binding at the MYC binding motif was analyzed by real-time PCR after JQ1 treatment $(0.2 \mu \mathrm{M})$ for $24 \mathrm{~h}$. Data are presented as the mean \pm SEM ${ }^{* * *} \mathrm{P}<0.001 \mathrm{vs}$. control). (G) A proposed model of MYC-AP4 axis repression by the BRD4 inhibitor JQ1 (refer to Results and Discussion for a detailed explanation). 
knockdown of AP4 induces downregulation of MYC. This may be the underlying mechanism by which suppression of the MYC-AP4 axis is amplified by the BET protein inhibitor. AP4 is also known to contribute to several processes during cancer development, including EMT and metastasis (6). Our study showed that JQ1 downregulated AP4 at a very early time point and at low concentrations in the ER-positive and -negative cancer cell lines (Figs. 1 and 2). These results suggest that AP4 is the most sensitive and critical target of BET protein inhibition. The clinical relevance of AP4 expression in cancers was previously reported. Elevated expression of AP4 is associated with an increased metastatic capacity in colorectal cancer (6) and AP4 predicts poor prognosis in non-small cell lung cancer (25). In addition, several studies have demonstrated the critical role of AP4 in cancers and in immunology (26-28). Therefore, this study contributes to our understanding of cancer biology.

It is well known that epigenetic modifiers and chromatin remodelers control gene expression and establish cellular identities via the regulation of chromatin structure. However, little is known concerning the specific targets of these components of the epigenome and the underlying mechanisms of the effects of the small molecules that target them. Our results showed, for the first time, that the BET protein inhibitor suppressed the MYC-AP4 axis by targeting a bidirectional loop between MYC and AP4 to induce antitumorigenic effects. Collectively, our results suggest that a better understanding of epigenetic modifiers and chromatin remodelers will facilitate the development of novel strategies for treating many diseases caused by dysregulated epigenome components.

\section{Acknowledgements}

This research was supported by the Basic Science Research Program through the National Research Foundation of Korea (NRF) funded by the Ministry of Science, ICT and Future Planning (2013R1A1A1057575).

\section{References}

1. You JS and Jones PA: Cancer genetics and epigenetics: Two sides of the same coin? Cancer Cell 22: 9-20, 2012.

2. Arrowsmith CH, Bountra C, Fish PV, Lee K and Schapira M: Epigenetic protein families: A new frontier for drug discovery. Nat Rev Drug Discov 11: 384-400, 2012.

3. You JS and Han JH: Targeting components of epigenome by small molecules. Arch Pharm Res 37: 1367-1374, 2014.

4. Filippakopoulos P and Knapp S: Targeting bromodomains: Epigenetic readers of lysine acetylation. Nat Rev Drug Discov 13 337-356, 2014

5. Meyer N and Penn LZ: Reflecting on 25 years with MYC. Nat Rev Cancer 8: 976-990, 2008.

6. Jackstadt R, Röh S, Neumann J, Jung P, Hoffmann R, Horst D, Berens C, Bornkamm GW, Kirchner T, Menssen A, et al: AP4 is a mediator of epithelial-mesenchymal transition and metastasis in colorectal cancer. J Exp Med 210: 1331-1350, 2013.

7. Jung P, Menssen A, Mayr D and Hermeking H: AP4 encodes a c-MYC-inducible repressor of p21. Proc Natl Acad Sci USA 105: 15046-15051, 2008.
8. Jung $\mathrm{P}$ and Hermeking H: The c-MYC-AP4-p21 cascade. Cell Cycle 8: 982-989, 2009.

9. Dang CV: MYC on the path to cancer. Cell 149: 22-35, 2012.

10. Asangani IA, Dommeti VL, Wang X, Malik R, Cieslik M, Yang R, Escara-Wilke J, Wilder-Romans K, Dhanireddy S, Engelke C, et al: Therapeutic targeting of BET bromodomain proteins in castration-resistant prostate cancer. Nature 510: 278-282, 2014.

11. Delmore JE, Issa GC, Lemieux ME, Rahl PB, Shi J, Jacobs HM, Kastritis E, Gilpatrick T, Paranal RM, Qi J, et al: BET bromodomain inhibition as a therapeutic strategy to target c-Myc. Cell 146: 904-917, 2011

12. Zuber J, Shi J, Wang E, Rappaport AR, Herrmann H, Sison EA, Magoon D, Qi J, Blatt K, Wunderlich M, et al: RNAi screen identifies Brd4 as a therapeutic target in acute myeloid leukaemia. Nature 478: 524-528, 2011.

13. Lockwood WW, Zejnullahu K, Bradner JE and Varmus H: Sensitivity of human lung adenocarcinoma cell lines to targeted inhibition of BET epigenetic signaling proteins. Proc Natl Acad Sci USA 109: 19408-19413, 2012.

14. Rakha EA, Reis-Filho JS and Ellis IO: Basal-like breast cancer: A critical review. J Clin Oncol 26: 2568-2581, 2008.

15. Ignatiadis M and Sotiriou C: Luminal breast cancer: From biology to treatment. Nat Rev Clin Oncol 10: 494-506, 2013.

16. Bihani T, Ezell SA, Ladd B, Grosskurth SE, Mazzola AM, Pietras M, Reimer C, Zinda M, Fawell S and D'Cruz CM: Resistance to everolimus driven by epigenetic regulation of MYC in $\mathrm{ER}^{+}$breast cancers. Oncotarget 6: 2407-2420, 2015

17. Stratikopoulos EE, Dendy M, Szaboles M, Khaykin AJ, Lefebvre C, Zhou MM and Parsons R: Kinase and BET inhibitors together clamp inhibition of PI3K signaling and overcome resistance to therapy. Cancer Cell 27: 837-851, 2015.

18. Huang MJ, Cheng YC, Liu CR, Lin S and Liu HE: A small-molecule c-Myc inhibitor, 10058-F4, induces cell-cycle arrest, apoptosis, and myeloid differentiation of human acute myeloid leukemia. Exp Hematol 34: 1480-1489, 2006.

19. Mo $\mathrm{H}$ and Henriksson M: Identification of small molecules that induce apoptosis in a Myc-dependent manner and inhibit Myc-driven transformation. Proc Natl Acad Sci USA 103: 6344-6349, 2006.

20. Belkina AC and Denis GV: BET domain co-regulators in obesity, inflammation and cancer. Nat Rev Cancer 12: 465-477, 2012.

21. Filippakopoulos P, Qi J, Picaud S, Shen Y, Smith WB, Fedorov O, Morse EM, Keates T, Hickman TT, Felletar I, et al: Selective inhibition of BET bromodomains. Nature 468: 1067-1073, 2010.

22. Lovén J, Hoke HA, Lin CY, Lau A, Orlando DA, Vakoc CR, Bradner JE, Lee TI and Young RA: Selective inhibition of tumor oncogenes by disruption of super-enhancers. Cell 153: 320-334, 2013.

23. Hnisz D, Abraham BJ, Lee TI, Lau A, Saint-André V, Sigova AA, Hoke HA and Young RA: Super-enhancers in the control of cell identity and disease. Cell 155: 934-947, 2013.

24. Hnisz D, Schuijers J, Lin CY, Weintraub AS, Abraham BJ, Lee TI, Bradner JE and Young RA: Convergence of developmental and oncogenic signaling pathways at transcriptional super-enhancers. Mol Cell 58: 362-370, 2015.

25. Gong H, Han S, Yao H, Zhao H and Wang Y: AP-4 predicts poor prognosis in non small cell lung cancer. Mol Med Rep 10: 336-340, 2014.

26. Chou C, Pinto AK, Curtis JD, Persaud SP, Cella M, Lin CC, Edelson BT, Allen PM, Colonna M, Pearce EL, et al: c-Myc-induced transcription factor AP4 is required for host protection mediated by CD8 ${ }^{+} \mathrm{T}$ cells. Nat Immunol 15: 884-893, 2014.

27. Jackstadt R and Hermeking H: AP4 is required for mitogen- and c-MYC-induced cell cycle progression. Oncotarget 5: 7316-7327, 2014.

28. Jackstadt R, Jung P and Hermeking H: AP4 directly downregulates p16 and p21 to suppress senescence and mediate transformation. Cell Death Dis 4: e775, 2013. 